MATEC Web of Conferences 32, 07005 (2015)

DOI: $10.1051 /$ matecconf/20153207005

C) Owned by the authors, published by EDP Sciences, 2015

\title{
Adaptive micro axicons for laser applications
}

\author{
Ulrike Wallrabe ${ }^{1, a}$, Jens Brunne ${ }^{1}$, Alexander Treffer ${ }^{2}$, and Ruediger Grunwald² \\ ${ }^{1}$ Laboratory for Microactuators, Department of Microsystems Engineering (IMTEK), University of Freiburg, Georges-Koehler-Allee 102, \\ 79110 Freiburg Germany \\ ${ }^{2}$ Max Born Institute for Nonlinear Optics and Short-Pulse Spectroscopy (MBI), Max Born Str. 2a, 12489 Berlin, Germany
}

\begin{abstract}
We report on the design, fabrication and testing of novel types of low-dispersion axicons for the adaptive shaping of ultrashort laser pulses. An overview is given on the basic geometries and operating principles of our purely reflective adaptive MEMS-type devices based on thermal or piezoelectric actuation. The flexible formation of nondiffracting beams at pulse durations down to a few oscillations of the optical field enables new applications in optical communication, pulse diagnostics, laser-matter interaction and particle manipulation. As an example, we show first promising results of adaptive autocorrelation. The combination of excellent pulse transfer, self-reconstruction properties and propagation invariance of nondiffracting beams with an adaptive approach promises to extend the field of practical applications significantly.
\end{abstract}

\section{Introduction}

With the commercial availability of ultrashort pulse lasers with pulse durations as short as a few femtoseconds and large bandwidths, science and industry get powerful tools for studying and controlling physical and chemical processes at extreme time scales and to realize ultrafast optical communication systems. Another, particularly important field of applications are biomedical treatment and analysis. All of these emerging developments, however, require not only the laser sources but also appropriate devices for shaping, guiding and diagnosing the laser radiation in space, time and spectrum. Therefore, the development of advanced beam-shaping components plays a key role. Hence, we run a long-standing research program and combine engineering, adaptive optics and laser physics which proved to be a fruitful approach to overcome the intrinsic limitations of conventional beam shapers. Here, we report on the state of the art of designing and realizing novel types of tunable axicons for ultrafast laser applications based on reflective micro-electromechanical systems (MEMS).

\section{Types of adaptive non-diffractive beam shapers for ultrashort pulses}

\subsection{Discrete and analogue profile functions}

Most of the previous work on adaptively shaping ultrashort laser pulses has been done with spatial light modulators (SLMs) and digital mirror devices (DMDs). In particular, liquid-crystal-on-silicon (LCOS) SLMs have been employed to enable the generation of an enormous variety of spatial and temporal features in ultrashort pulses, e.g. Bessel beams or Bessel-like needle beams [1], highly localized wavepackets [2] and vortex beams [3]. A pulse transfer was obtained down to pulse durations of about 6.5 fs [3] by compensating the dispersion.

Until now, the clear advantage of such components has been their superior structural flexibility which results from the large number of sub-apertures (up to millions of pixels). On the other hand, the pixelated structure reduces the fill factor and introduces diffraction effects. The light transmission through liquid crystal layers causes dispersive pulse lengthening and absorption and is thus a limiting factor with respect to pulse duration and damage

\footnotetext{
${ }^{\mathrm{a}}$ Corresponding author: wallrabe@ imtek.uni-freiburg.de
}

resistance. Essential improvements are expected, however, for flexible metal-coated continuous surface structures. Their continuous reflecting surfaces induce less parasitic diffraction effects and enable to minimize chirp by reduced dispersion. Selected types of continuous MEMS axicons will be reported in more detail in paragraph 3 .

\subsection{Tuning principles}

After comparing optional MEMS designs, we decided to develop two main variants of MEMS axcions: (a) one based on piezoelectric actuation and (b) one based on thermal actuation. Both types of elements consist of layered structures coated with a reflecting layer, each of which featuring its own intrinsic advantages and disadvantages. Type-(a)-axicons are constructed of a thin silicon plate coated with aluminium which is mounted onto a piezo bending actuator. They can be used for high-speed switching (up to few-kHz-range) but display a hysteresis effect, whereas type-(b)-axicons are quite slow and only tunable on a second scale.

\section{Overview on realized types of MEMS axicons}

Figure 1 shows an overview on the main types of MEMS axicon geometries and arrangements which were realized up to now. Starting from a single circular axicon (Fig. 1 a) with both piezoelectric [4] and thermal actuation [5], an array of thermal micro axicons was produced for the adaptive generation of Bessel-like beams (Fig. 1 c) (patent pending). A Fresnel bi-mirror or "linear axicon" (Fig. 1b) was actuated by a piezobending actuator [6] to adaptively shape light blades. The process for the thermally actuated devices proved to be amazingly flexible and thus allowed for the unique fabrication of a spiral phase plate (SPPs, Fig. 1 d) for the generation of vortex beams carrying an orbital angular momentum $(\mathrm{OAM})$ of tunable topological charge [7].

Figure 1 (a) to (d) shows the surface profiles of various MEMS axicon types obtained with a white light interferometer Typical maximum phase strokes were in a few micrometer range and correspond to maximum conical angles in the range of a few degrees. 


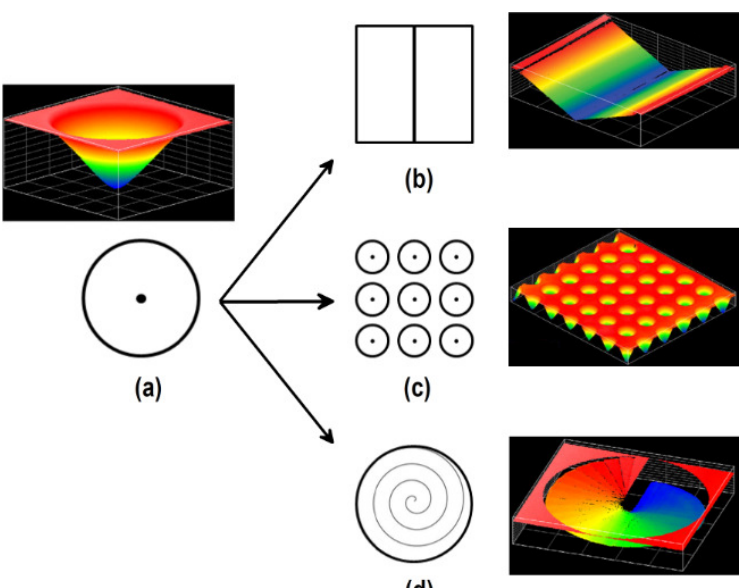

(d)

Figure 1. Family tree of MEMS axicons: (a) circular axicon (type a and b), (b) linear axicon/Fresnel's bi-mirror (type a), (c) simultaneously tuned array of circular axicons (type b), and (d) spiral phase plate (type b). The coloured plots represent white light interferometric measurements of the surface profiles.

Figure 2 shows as an example the fabrication, working principle, and a micrograph of a thermally tuned $2 \times 2$ axicon array. First, we structured a silicon mold corresponding to the desired conical shape by direct laser ablation and cleaned it in $\mathrm{KOH}$ resulting in a surface roughness of $1 \mu \mathrm{m} \mathrm{R}_{\mathrm{a}}$. We then dispensed an adhesion promoter (Dow Corning 92-023) followed by liquid polydimethylsiloxane (PDMS, Sylgard 184) into the mold. We placed the chip into an alignment mount and pressed a second chip, which carried the reflective Al layer as well as a thin etch-stop and adhesion layers, on top of it. The PDMS was subsequenty cured under vacuum at $59^{\circ} \mathrm{C}$, while keeping the pressure between the two chips constant. Due to the curing time at that temperature of more than 5 min. [8], the liquid PDMS expanded due to thermal expansion and excess material was pressed out of the mold. After $3 \mathrm{~h}$, the polymer was post-cured at $120^{\circ} \mathrm{C}$ for $45 \mathrm{~min}$.

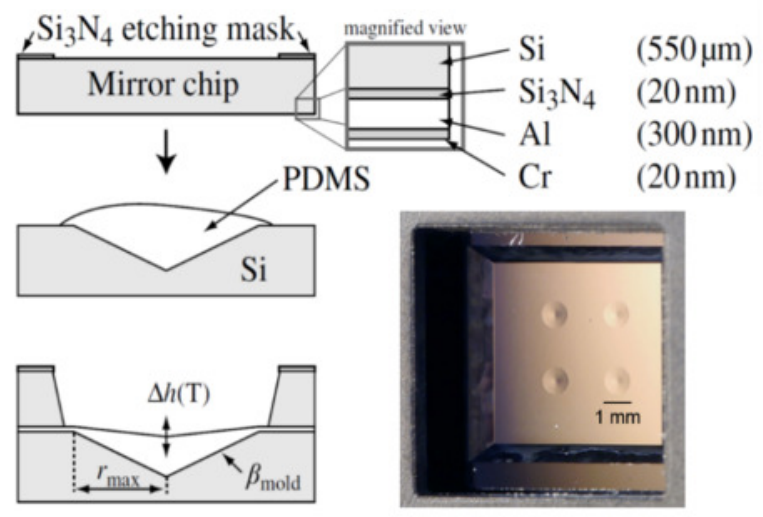

Figure 2. Fabrication and working principle of thermally tunable micro axicon array. Element diameter is $1 \mathrm{~mm}$.

The Al mirror layer indicated in Fig. 2 then was exposed by etching a window through the upper silicon chip with $30 \% \mathrm{KOH}$ at $80^{\circ} \mathrm{C}$. Now, as the rigid top layer had been removed, the stress that had been induced in the PDMS due to volume shrinkage was released. The PDMS shrank accordingly, thus adapting to the conical shape of the silicon mold, but at a much smaller angle. The roughness of the mold was thereby smoothed by several orders of magnitude and resulted in an optical surface with a roughness of $R_{a}<6 \mathrm{~nm}$. Finally, the axicon array was placed on a custom made chip mount with integrated heating and cooling, and the angle of the axicons could be controlled via the temperature.

\section{Beam shaping and applications}

\subsection{Fresnel bi-mirrors}

The Fresnel bi-mirrors consist of two partial mirrors on piezo-actuators which undergo a synchronous tilt movement (like butterfly wings) [6]. By the superposition of both partial beams, this leads to the formation of "light blades" - extended (quasi-nondiffracting) linear focal zones [9]. Their number, thickness and length can be tuned by adjusting the tilt angle of the mirrors.

Application experiments were performed to generate variable excitation zones for laser-induced periodic surface structures (LIPSS) [10] and to study the adaptive autocorrelation of few-cycle pulses [11]. In this case, the self-referential interferometric setup (known from inducing holographic gratings) in combination with a thin nonlinear crystal (beta barium borate, BBO) detects the second order autocorrelation function, thus acting as a non-collinear autocorrelator. In contrast to conventional autocorrelators, angular tuning enables for an optimization by adaptive scaling and minimizing the influence of local distortions ("adaptive self-reconstruction"). As an example, the non-collinear autocorrelation traces for two different tilt angles of a linear axicon are compared to each other in Fig. 3.
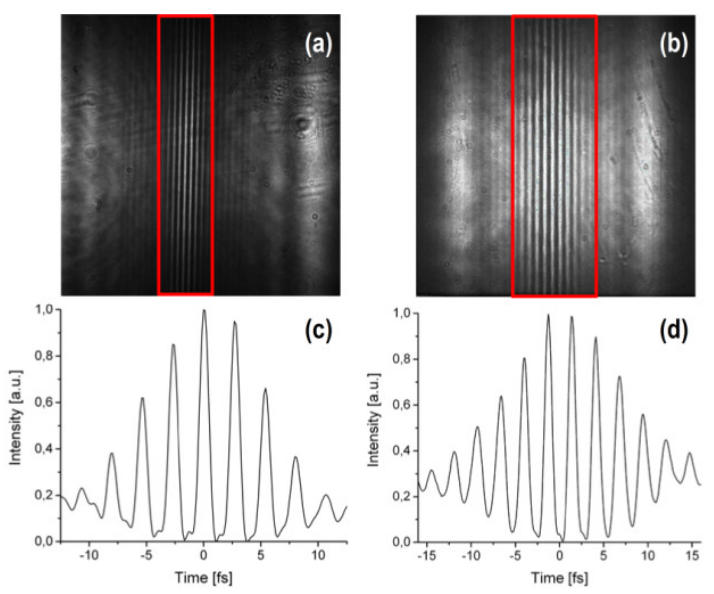

Figure 3. Adaptive non-collinear second order autocorrelation of few-cycle pulses with scaling optimization, $(\mathrm{a}, \mathrm{c})$ incomplete overlap of the two partial beams, (b,d) nearly complete overlap (closest approximation of the pulse duration).

The obtaineded FWHM time duration of the autocorrelation function in $(\mathrm{a}, \mathrm{c})$ is unrealistically short because of a spatial cut due to incomplete spatial superposition. Incontrast, the spatial superposition in $(b, d)$ is nearly complete and delivers a pulse duration close to the value expected by reference measurements with spectral phase interferometry by direct electric-field reconstruction (SPIDER) [9]. The corresponding pulse duration for the used configuration was found to be about $8 \mathrm{fs}$. 


\subsection{Circular axicons}

Circular thermally tuned axicons were applied to generate adaptive Bessel-like beams [4] which can be used, e.g., for flexible materials processing or filament generation. The extension of the depth of the focus zone by changing the temperature of the actuator (corresponding to a change of the conical angle) is shown in Fig. 4 for 3 distinct temperatures. In this interval, the Rayleigh length increases by a factor of about 5 .

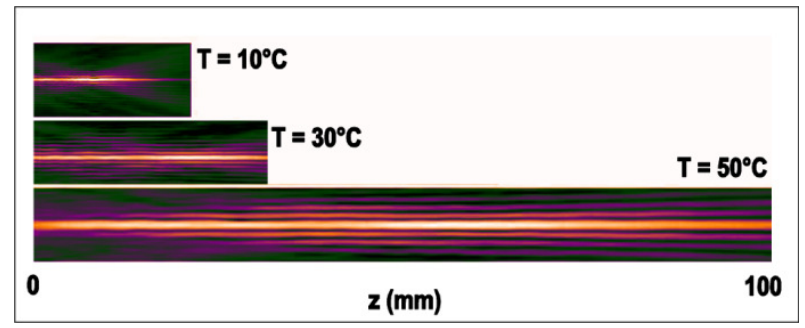

Figure 4. Tuning the focal zone of a quasi-Bessel beam with a thermally driven axicon. The beam propagation is shown for three selected temperatures (thermalized state) [4].

\subsection{Spiral phase plates}

We used the thermally driven spiral phase plates shown in fig. $1 \mathrm{~d}$ to generate 7 -fs vortex pulses with continuously tunable topological charge [7] as shown in Figure 5with a continuous phase profile of up to $14 \pi$. Compared to alternative solutions like high-power optical parametric oscillators (OPOs) as shapers, our approach allows us to work with much more compact systems. This can be an enormous advantage for practical applications, e.g., in optical communication systems or data processing.
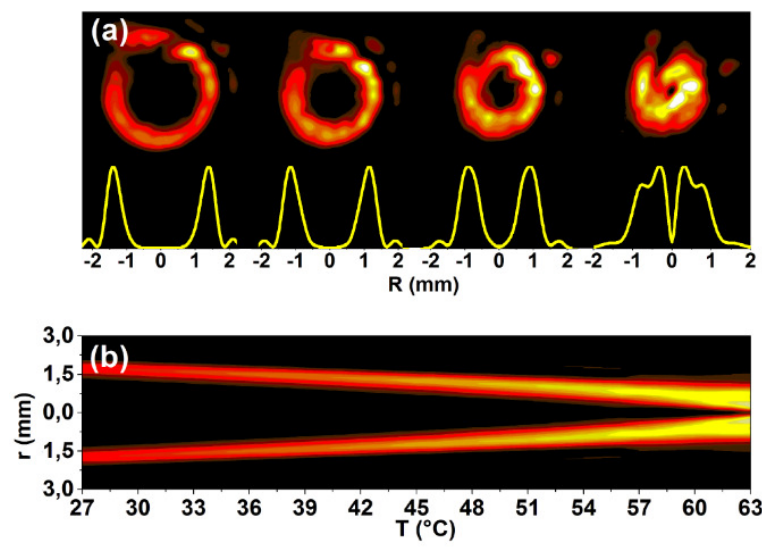

Figure 5. Tunability of the few-cycle vortex beams: (a) measured light distributions recorded at a distance of $750 \mathrm{~cm}$ at an incident angle of $20^{\circ}$ indicating topological charges of 7, 5, 3, and 1 (from left to right) and (b) beam distribution as a function of temperature. The oscillations are suppressed due to averaging.

\section{Conclusions and outlook}

We have developed, characterized and applied novel types of purely reflective MEMS axicons for shaping ultrashort-pulsed nondiffracting beams. These devices with apertures between 1 and $10 \mathrm{~mm}$ enable the generation of tunable Bessel-like beams, line foci and vortex beams by adjusting the conical angles or the phase stroke of spiral phase shapers (thus varying the topological charge). This was realized for single elements and arrays. While the circular axicons and spiral phase plates were thermally controlled, Fresnel bi-mirrors were driven by piezoactuators. We demonstrated the capability of MEMS axicons to shape and transfer sub-3-cycle pulses at $800 \mathrm{~nm}$ central wavelength in first proof-of-principle experiments. We were also able to shape variable multichannel excitation zones and - for the first time - the non-collinear autocorrelation of ultrashort pulses with adaptive scaling and self-reconstruction.

Currently, we investigate array-specific effects induced by multiple MEMS arrangements and variable surface plasmon excitation. Furthermore, tunable reflective MEMS axicons are promising candidates for new phase-shifting approaches in interferometry, autocorrelation, digital holography and complex phase retrieving pulse characterization techniques.

Besides these pure technical applications, Bessel beams came into focus for their beneficial use in life sciences. Once, it was shown that they are "selfreconstructing" [12] and show enhanced penetration into scattering media [13], they are increasingly investigated in microscopy [14]. Currently, they are explored for their application in neurosciences for opto-genetic stimulation.

The authors acknowledge discussions and essential contributions by M.C. Wapler (IMTEK), and T. Elsaesser, G. Steinmeyer, M. Bock, S. König (MBI). The work was financially supported by DFG (grants WA 11657/3-1 and GR 1782/14-1).

\section{References}

1. M. Bock, R. Grunwald, Appl. Sci. 3, 139-152 (2013).

2. M. Bock, S. K. Das, R. Grunwald, Opt. Express 20, 12563-12578 (2012).

3. R. Grunwald, T. Elsaesser, M. Bock, Sci. Rep. 4, 07148 (2014).

4. J. Brunne, U. Wallrabe, Opt. Lett. 38, 1939-1941 (2013).

5. J. Brunne, M.C. Wapler, U. Wallrabe, Opt. Lett. 39, 4631-4634 (2014)

6. J. Brunne, M. Wapler, R. Grunwald, U. Wallrabe, J. Micromech. Microeng. 23, 115002 (2013).

7. M. Bock, J. Brunne, A. Treffer, S. König, U. Wallrabe, R. Grunwald, Opt. Lett. 38, 3642-3645 (2013).

8. F. Schneider, J. Draheim, R. Kamberger, U. Wallrabe, Sens. Actuators A 151, 95 (2009).

9. A. Treffer, S. K. Das, M. Bock, J. Brunne, U. Wallrabe, R. Grunwald, Proc. SPIE 8637, 86370M (2013).

10. A. Treffer, S. K. Das, H. Messaoudi, J. Brunne, U. Wallrabe, R. Grunwald, PIERS, Stockholm (2013).

11. A. Treffer, S. König, M. Bock, J. Brunne, U. Wallrabe, R. Grunwald, Proc. SPIE 9379, 9379-2 (2015).

12. F.O. Fahrbach, A. Rohrbach, Nature Communications 3, 632 (2012)

13. F.O.Fahrbach, V. Gurchenkov, K. Alessandri, P. Nassoy, A. Rohrbach, Opt. Express 21, no. 9, 11425 (2013)

14. T. Planchon, E. Belzig, Nature Methods 8 (2011), 417 